\title{
Plant Humanities Lab: Planting the seeds for creative data visualisation
}

\author{
Ashley Buchanan \\ Dumbarton Oaks \\ Washington, DC USA \\ buchanana01@doaks.org
}

\author{
Ron Snyder \\ ITHAKA \\ Ann Arbor, Michigan USA \\ Ron.Snyder@ithaka.org
}

\section{INTRODUCTION}

The Plant Humanities Lab is an innovative digital space that supports the interdisciplinary study of plants from the various perspectives of the arts, sciences, and humanities, to explore their extraordinary significance to human culture.

The Plant Humanities (https://lab.planthumanities.org/) is an open access and open-source prototype developed collaboratively by Dumbarton Oaks, a Harvard University research institute, and JSTOR Labs as part of the Plant Humanities Initiative. The Plant Humanities Lab provides a venue for students and the public to visually explore the cultural histories of plants through interconnected data from primary and secondary sources such as JSTOR Global Plants, the Biodiversity Heritage Library (BHL), and more. The Plant Humanities Lab also provides a set of online tools for students and researchers in the emerging field of Plant Humanities.

The first, and current, version includes 2 core tools, both powered by knowledge graphs and linked open data (LOD) technology.

- A tool for creating and displaying interactive visual narratives

- A semantic search tool for discovering secondary and primary resources associated with a named entity

With its roots in the early modern period, botany has always been a highly visual science and the Plant Humanities Lab allows for the integration of a massive and visually engaging repository of botanical art, illustrations, and herbaria. A key element of Plant Humanities Lab architecture is its

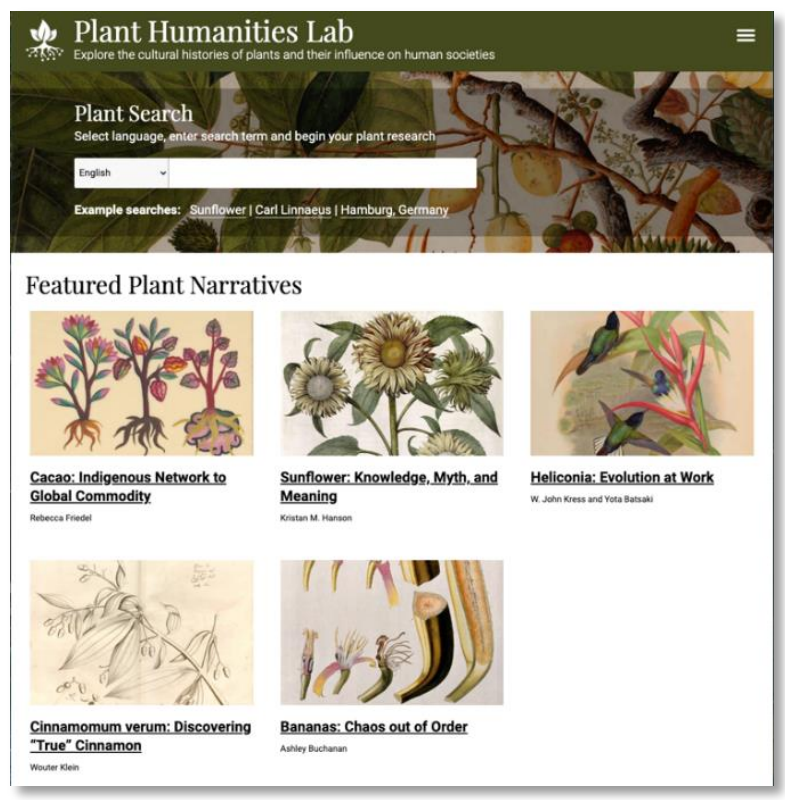

Figure 1: Plant Humanities Lab

integration with Wikidata, a large open-source knowledge graph used by Wikimedia projects like Wikipedia. Information from Wikidata can be easily utilized for data visualizations.

\section{VISUAL NARRATIVES}

Utilizing BHL, Global Plants, and other open access digitized resources, an interdisciplinary and intergenerational team of scholars created visually engaging and interactive narratives of plants across historical periods and cultures. Take for example the banana, which was first domesticated 7,000 years ago. Annotated diagrams explain the science and morphology of domesticated bananas, while an interactive map moves the user through the fruit's 
migration from the Philippines and Papua New Guinea to Indonesia, China, India, East Africa, and eventually Europe and the New World. The naming, understanding, and cultural significance of bananas is then traced through IIIF images of early modern travel accounts, herbals, and florilegium digitized from BHL.

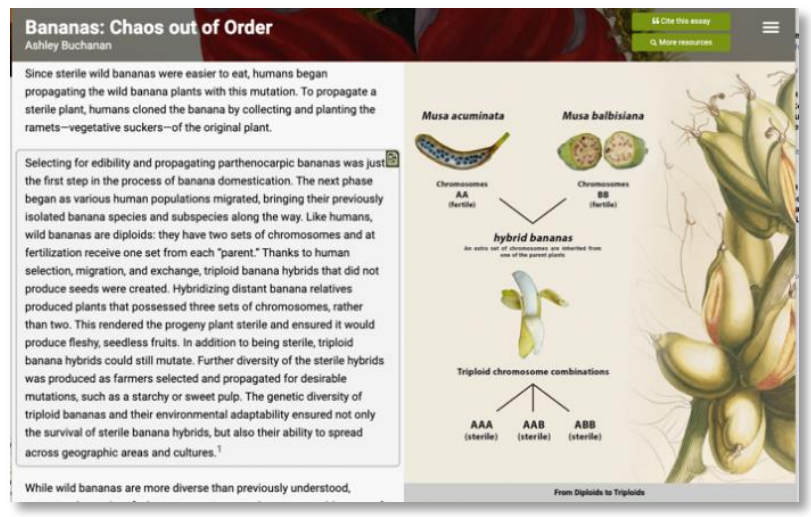

Figure 2: Annotated diagram in Banana visual narrative

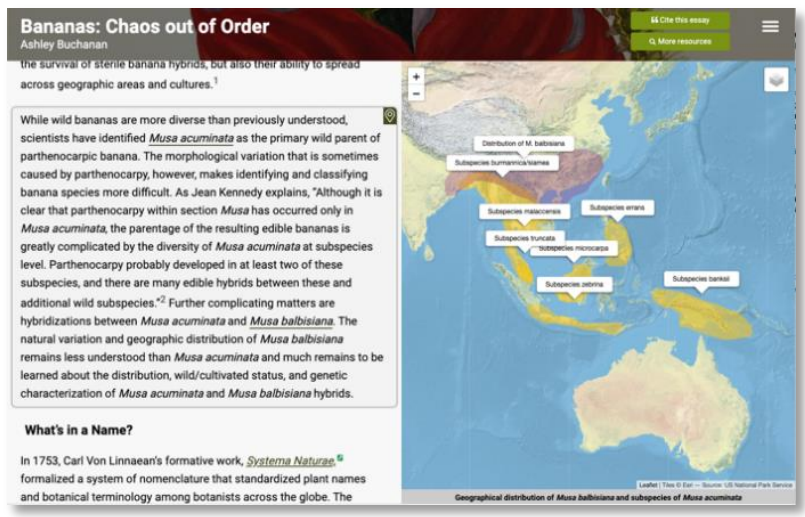

Figure 3: Interactive map in Banana visual narrative

Within the visual narratives, Wikidata is used to explain, elaborate, or connect to larger concepts or themes within each narrative. For example, in the visual narrative on dittany, Wikidata provides definitions for historic and scientific concepts the reader maybe unfamiliar with, while also connecting contemporary taxonomic information to historic plant names, identifications, and uses.

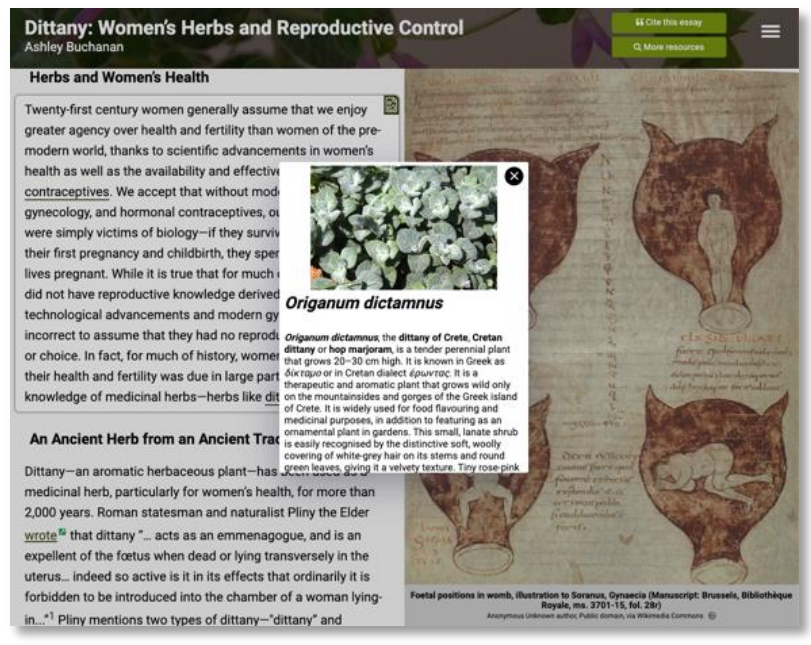

Figure 4: Dittany visual narrative with infobox
The data available through Wikidata also allows researchers to visualize networks, timelines, and time-encoded maps. For example, an interactive D3 generated network powered by Wikidata QIDs in the visual narrative on Heliconia demonstrates the close relationship between heliconias and their pollinators and highlights the vital role these plants play in the broader ecosystem as a source of food and shelter. And in the essay on boxwood, a time-encoded map visualizes the rapid pace at which "boxwood blight" invaded North America and threatened an entire species.

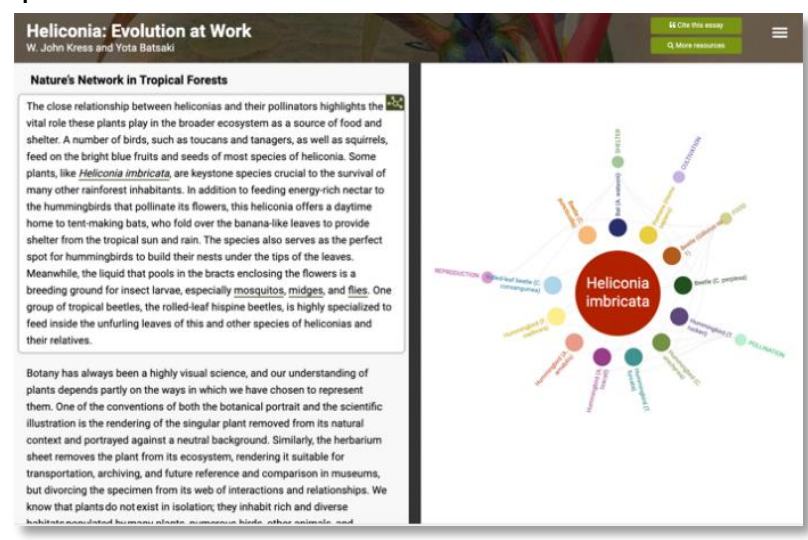

Figure 5: Interactive D3 network visualization from Heliconia visual narrative

The stories presented In the Plant Humanities Lab, all of which have been peer reviewed, showcase the global mobility of plants in the context of their economic, aesthetic, medicinal, culinary, political, and cultural significance. Users can follow these guided interactive tours, or explore the many resources offered as starting points for their own explorations.

The goal of these visual narratives is to highlight the significance of plants to human cultures via text paired with maps, high-resolution interactive IIIF (International Image Interoperability Framework) images, data visualizations, videos, and other multimedia. The Plant Humanities Lab not only allows users to visually explore the history and significance of an individual plant, but it also provides curated primary and secondary resources as well as embedded visual essays tools, which allows users to begin researching and creating their own visual narrative.

\section{SEMANTIC SEARCH}

The semantic search tool is both a companion to the visual narratives tool and a powerful entitybased search engine usable as a standalone research tool. The search tool federates community-curated data from the Wikidata and JSTOR knowledge graphs in a multilingual interface that performs search term disambiguation using autocomplete.

The result of a search is information for a single entity (person, location, concept, etc.) with 
structured data obtained from the Wikidata and JSTOR knowledge graphs. The knowledge graph data is augmented with other data obtained from external sources such as BHL, Global Plants, Artstor, Wikimedia Commons, and JSTOR. The search tool combines secondary and primary data sources providing a rich source of information for visual essay creators and general research.

Below is an example depicting results for sunflower (or Helianthus annuus) which includes structured data from Wikidata and links to primary and secondary sources from providers including Artstor, $\mathrm{BHJ}$, and JSTOR. The tool architecture is extensible and other content sources will be added in future versions.

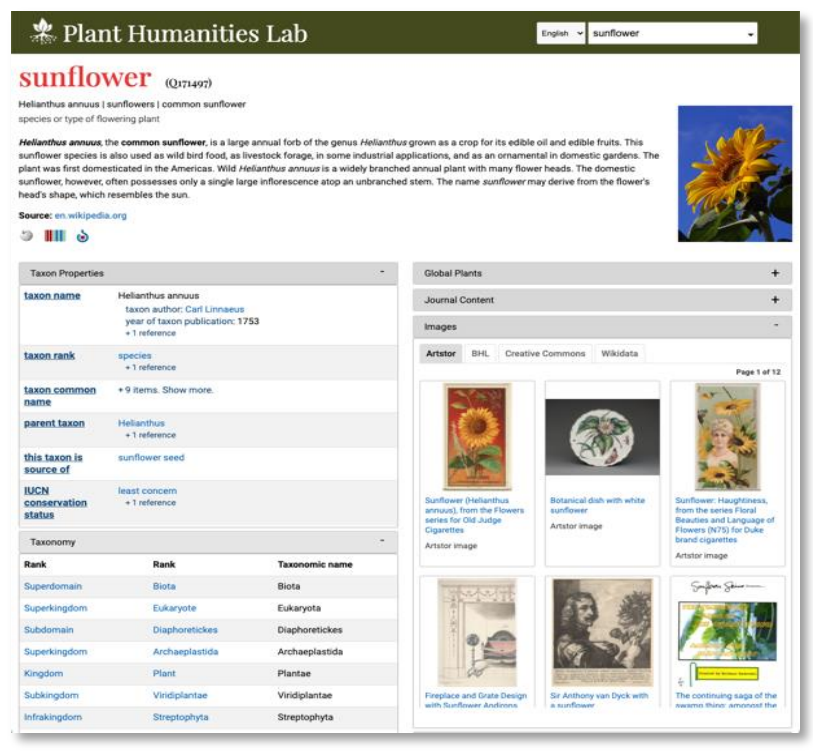

Figure 6: Semantic search results for Sunflower

\section{BEYOND PLANTS}

The capabilities developed for the Plant Humanities Lab are available for general use in a suite of opensource tools named Juncture. Using Juncture with the free Github site users and organizations can quickly create single narratives or a complete site such as the Plant Humanities Lab with free tools requiring little to no technical background to get started. No special tools are required as narratives are generated from plain text files annotated with simple tags for text formatting and visualization definitions.

\section{ABOUT THE PLANT HUMANITIES INITIATIVE}

In September 2018, Dumbarton Oaks and JSTOR Labs received grants from the Andrew W. Mellon Foundation to develop a digital platform and scholarly programming to advance the field of plant humanities. Bringing together humanists and digital technology experts, the Plant Humanities Lab was developed collaboratively by Dumbarton
Oaks and JSTOR Labs as a component of the Plant Humanities Initiative.

The plant narratives presented in the Plant Humanities Lab emerge from the interests of an interdisciplinary team whose members span various career stages, from doctoral and postdoctoral researchers and undergraduates to graduate summer participants of the Dumbarton Oaks Plant Humanities Initiative. As we add more stories, data, and additional features, we will continue to strive for a broad range of perspectives, historical periods, and geographies.

\subsection{Dumbarton Oaks}

Dumbarton Oaks (http://www.doaks.org/) is a research institute, museum, and historic garden of Harvard University. Founded in 1940 and located in Washington, DC, Dumbarton Oaks supports advanced research in Byzantine, Pre-Columbian, and Garden and Landscape Studies through fellowships, publications, and scholarly events. Dumbarton Oaks also welcomes the public to the museum, garden, concerts, and a rich program of lectures and conferences. The Plant Humanities Initiative (https://www.doaks.org/research/melloninitiatives/plant-humanities-initiative/) funded by the Andrew W. Mellon Foundation, draws on the riches of the rare book collection that is particularly strong in garden history, landscape architecture, botanical illustration, and plant history. In addition to the Plant Humanities Lab developed in collaboration with JSTOR Labs, the Plant Humanities Initiative encompasses post-doctoral and academic-year fellowships, a summer program with a digital skillbuilding component, publications, study days, an exhibition of botanical art (www.doaks.org/visit/museum/exhibitions), and a conference in 2022.

\subsection{JSTOR}

JSTOR Labs (http://labs.jstor.org/) partners with the scholarly community to develop and incubate experimental tools that expand access to knowledge. JSTOR Labs employs an agile, iterative, and user-focused approach that is inspired by the "design thinking" and "lean startup" communities. The team has employed these methods to develop tools like Text Analyzer (http://jstor.org/analyze), which helps users to autotext-mine pieces of writing (whether a published article or a user's draft paper) and discover relevant scholarly writings, and The JSTOR Understanding Series (http://jstor.org/understand), which maps and connects each line or passage from often-taught primary texts to all the scholarly articles quoting that line. Working with JSTOR Global Plants, the team also developed Livingstone's Zambezi Expedition (http://labs.jstor.org/projects/livingstones-zambeziexpedition/), which was one of the inspirations for the Plant Humanities Initiative. 